Supporting Information for

\title{
The influence of the composition of eight-atom Pt-Ir clusters on the magnetic properties
}

\author{
Nengwen Long ${ }^{\mathrm{a}}$, Jiguang $\mathrm{Du}^{\mathrm{b}}$, and Gang Jiang ${ }^{\mathrm{a}^{*}}$ \\ ${ }^{a}$ Institute of Atomic and Molecular Physics, Sichuan University, Chengdu, Sichuan 610065, China. \\ ${ }^{b}$ College of Physical Science and Technology, Sichuan University, Chengdu, Sichuan 610064, China.
}

\section{Supplemental materials:}

1. Table S1. Geometries, total magnetic moments, binding energies per atom and relative energies of four most stable isomers in the clusters.

2. Table S2. In $\mathrm{Pt}_{8-} \mathrm{Ir}_{n}$ clusters, electron distribution numbers and charge numbers of all the atoms after the optimization are summarized as follows.

3. Table S3. Local magnetic moments in the PAW sphere of both Pt and Ir atoms along with the spin population numbers in different orbitals are provided below.

4. Figure S1. Most favorable geometrical structures of the low-lying isomers for different compositions at a given size.

\footnotetext{
* Corresponding author at: Institute of Atomic and Molecular Physics, Sichuan University, Chengdu 610065, P. R. China. Tel.: +86 02885408810

E-mail address: gjiang@scu.edu.cn
} 
Table S1. Geometries, total magnetic moments (unit: $\mu_{\mathrm{B}}$ ), binding energies (unit: $\mathrm{eV} /$ atom) and relative energies (unit: $\mathrm{eV}$ ) of four most stable isomers in the clusters.

\begin{tabular}{|c|c|c|c|c|}
\hline Notation & Structure & $\begin{array}{c}\text { Total } \\
\text { Moment }\end{array}$ & $\begin{array}{l}\text { Binding } \\
\text { Energy }\end{array}$ & $\begin{array}{l}\text { Relative } \\
\text { Energy }\end{array}$ \\
\hline${ }^{1} \mathrm{~T}_{0}$ & & 4.37 & 3.490 & 0 \\
\hline${ }^{2} \mathrm{P}_{0}$ & & 4.00 & 3.464 & 0.210 \\
\hline${ }^{3} \mathrm{P}_{0}$ & & 3.99 & 3.460 & 0.243 \\
\hline${ }^{4} \mathrm{~T}_{0}$ & & 8.00 & 3.447 & 0.343 \\
\hline${ }^{1} \mathrm{~T}_{1}$ & & 3.62 & 3.704 & 0 \\
\hline${ }^{2} \mathrm{~T}_{1}$ & & 3.00 & 3.683 & 0.164 \\
\hline${ }^{3} \mathrm{~T}_{1}$ & & 6.99 & 3.669 & 0.282 \\
\hline${ }^{4} \mathrm{~T}_{1}$ & & 2.99 & 3.667 & 0.294 \\
\hline${ }^{1} \mathrm{~T}_{2}$ & & 4.03 & 3.902 & 0 \\
\hline
\end{tabular}




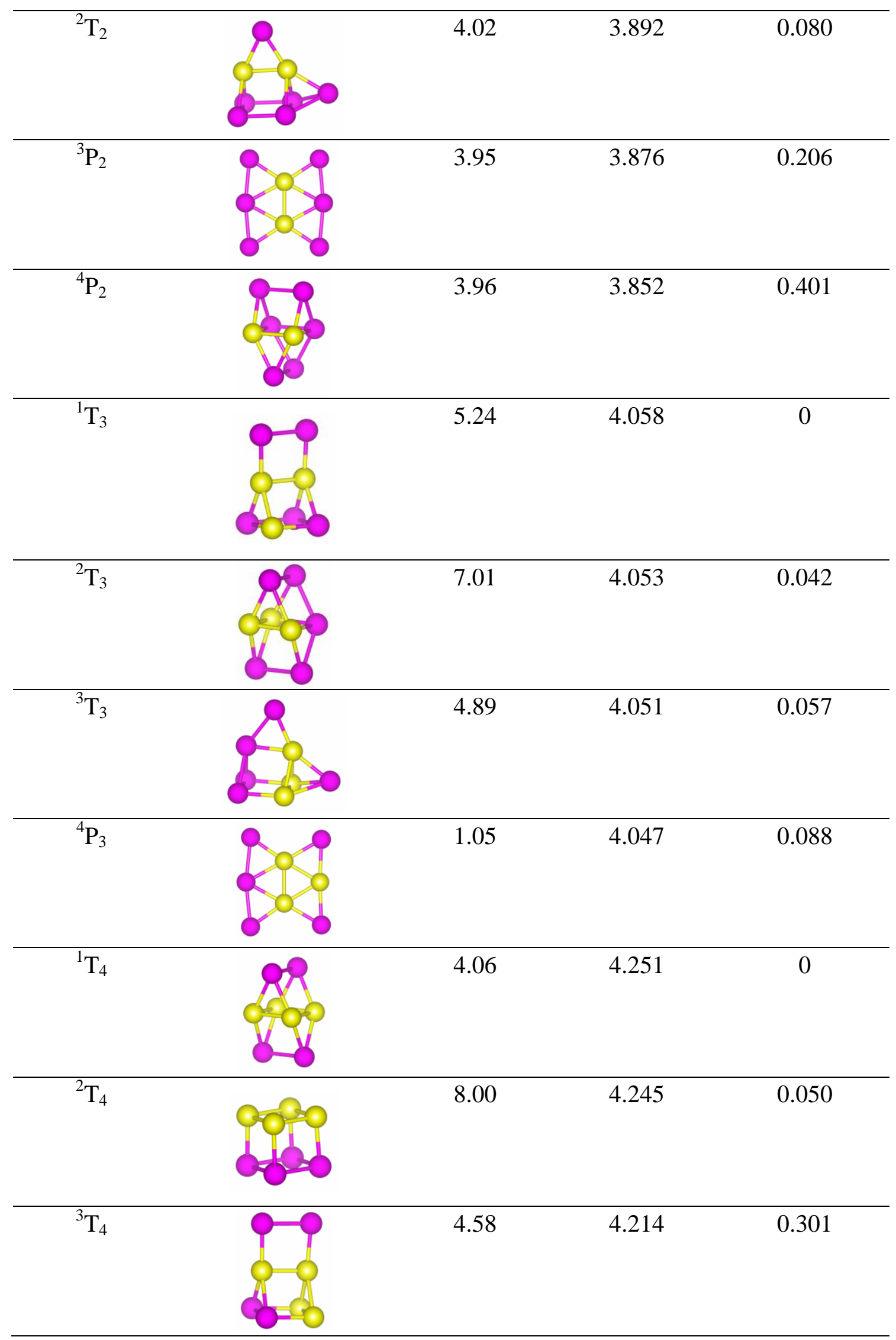




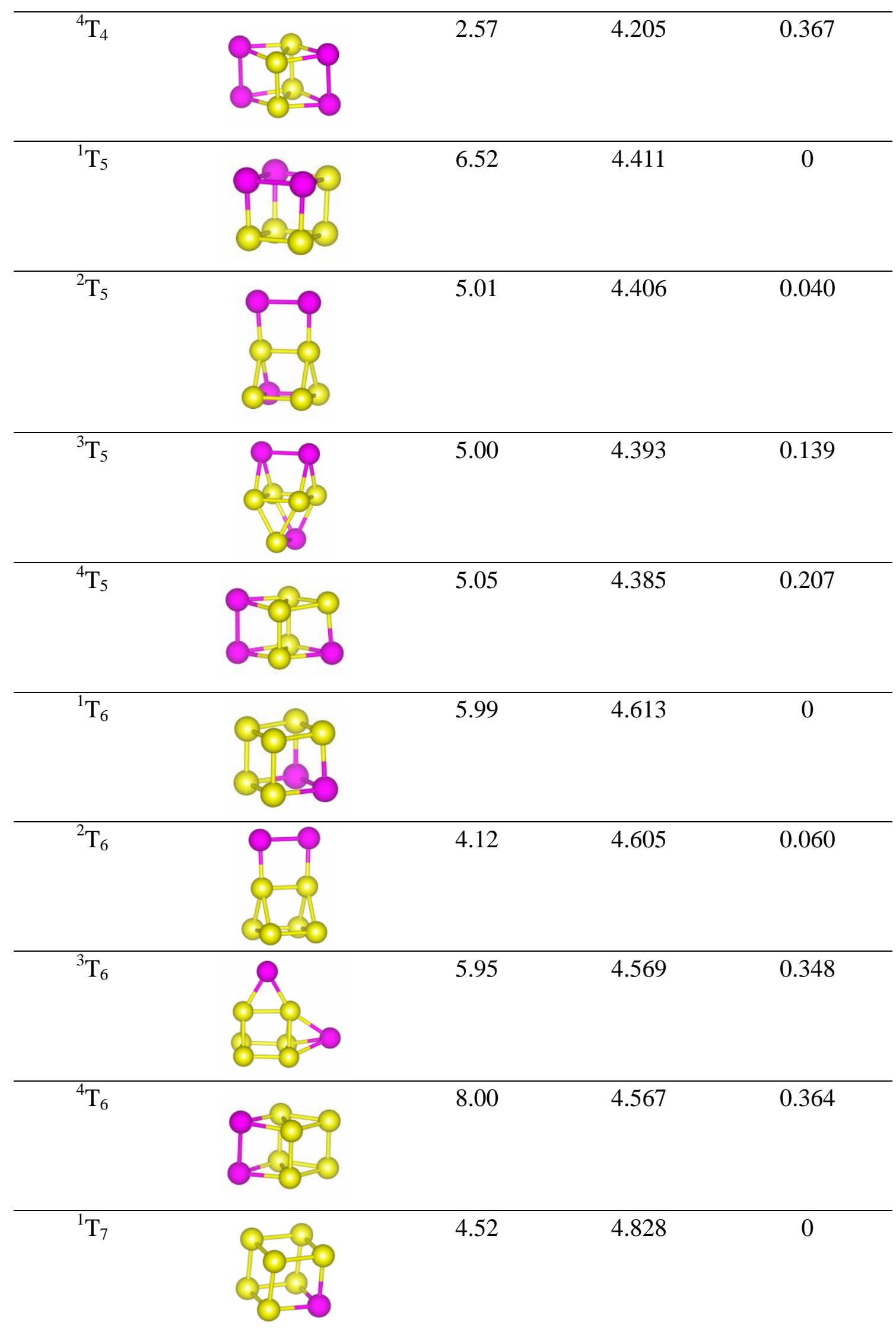




\begin{tabular}{|c|c|c|c|}
\hline${ }^{2} \mathrm{~T}_{7}$ & 4.84 & 4.720 & 0.868 \\
\hline${ }^{3} \mathrm{~T}_{7}$ & 10.69 & 4.700 & 1.027 \\
\hline${ }^{4} \mathrm{~T}_{7}$ & 7.23 & 4.668 & 1.283 \\
\hline${ }^{1} \mathrm{~T}_{8}$ & 0 & 5.114 & 0 \\
\hline${ }^{2} \mathrm{~T}_{8}$ & 8.00 & 4.895 & 1.759 \\
\hline${ }^{3} \mathrm{~T}_{8}$ & 0.20 & 4.876 & 1.906 \\
\hline${ }^{4} T_{8}$ & 11.83 & 4.846 & 2.147 \\
\hline
\end{tabular}

Notes: Notation ${ }^{\mathrm{i}} \mathrm{P}_{\mathrm{j}}$ or ${ }^{\mathrm{i}} \mathrm{T}_{\mathrm{j}}$ represents the corresponding geometry of clusters, where superscript $i$ shows energy order of clusters, and subscript $j$ means the atomic number of iridium atoms among the cluster as well, what's more, notation $\mathrm{P}$ and $\mathrm{T}$ represent the planar and three-dimensional structures, respectively. 
Table S2. In $\mathrm{Pt}_{8-n} \mathrm{Ir}_{n}$ clusters, electron distribution numbers and charge numbers of all the atoms after the optimization are summarized as follows.

\begin{tabular}{|c|c|c|c|}
\hline Clusters & Atoms (no.) & Electron distribution numbers & $\mathrm{Q}(\mathrm{e})$ \\
\hline \multirow[t]{8}{*}{$\mathrm{Pt}_{8}$} & Pt 1 & 9.964 & 0.036 \\
\hline & Pt 2 & 9.947 & 0.053 \\
\hline & Pt 3 & 9.925 & 0.075 \\
\hline & Pt 4 & 9.922 & 0.078 \\
\hline & Pt 5 & 10.064 & -0.064 \\
\hline & Pt 6 & 10.059 & -0.059 \\
\hline & Pt 7 & 10.042 & -0.042 \\
\hline & Pt 8 & 10.077 & -0.077 \\
\hline \multirow[t]{8}{*}{$\mathrm{Pt}_{7} \mathrm{Ir}$} & Ir 1 & 8.568 & 0.432 \\
\hline & Pt 2 & 10.250 & -0.250 \\
\hline & Pt 3 & 9.941 & 0.059 \\
\hline & Pt 4 & 10.063 & -0.063 \\
\hline & Pt 5 & 10.051 & -0.051 \\
\hline & Pt 6 & 9.972 & 0.028 \\
\hline & Pt 7 & 9.959 & 0.041 \\
\hline & Pt 8 & 10.196 & -0.196 \\
\hline \multirow[t]{8}{*}{$\mathrm{Pt}_{6} \mathrm{Ir}_{2}$} & Ir 1 & 8.708 & 0.292 \\
\hline & Ir 2 & 8.731 & 0.269 \\
\hline & Pt 3 & 10.049 & -0.049 \\
\hline & Pt 4 & 10.104 & -0.104 \\
\hline & Pt 5 & 10.115 & -0.115 \\
\hline & Pt 6 & 10.062 & -0.062 \\
\hline & Pt 7 & 10.096 & -0.096 \\
\hline & Pt 8 & 10.135 & -0.135 \\
\hline \multirow[t]{8}{*}{$\mathrm{Pt}_{5} \mathrm{Ir}_{3}$} & Ir 1 & 8.792 & 0.208 \\
\hline & Ir 2 & 8.676 & 0.324 \\
\hline & Ir 3 & 8.918 & 0.082 \\
\hline & Pt 4 & 10.046 & -0.046 \\
\hline & Pt 5 & 10.108 & -0.108 \\
\hline & Pt 6 & 10.139 & -0.139 \\
\hline & Pt 7 & 10.151 & -0.151 \\
\hline & Pt 8 & 10.169 & -0.169 \\
\hline \multirow[t]{8}{*}{$\mathrm{Pt}_{4} \mathrm{Ir}_{4}$} & Ir 1 & 8.762 & 0.238 \\
\hline & Ir 2 & 8.915 & 0.085 \\
\hline & Ir 3 & 8.835 & 0.165 \\
\hline & Ir 4 & 8.842 & 0.158 \\
\hline & Pt 5 & 10.125 & -0.125 \\
\hline & Pt 6 & 10.180 & -0.180 \\
\hline & Pt 7 & 10.143 & -0.143 \\
\hline & Pt 8 & 10.198 & -0.198 \\
\hline $\mathrm{Pt}_{3} \mathrm{Ir}_{5}$ & Ir 1 & 8.891 & 0.109 \\
\hline
\end{tabular}




\begin{tabular}{|c|c|c|c|}
\hline & Ir 2 & 8.887 & 0.114 \\
\hline & Ir 3 & 8.998 & 0.002 \\
\hline & Ir 4 & 8.909 & 0.091 \\
\hline & Ir 5 & 8.937 & 0.063 \\
\hline & Pt 6 & 10.134 & -0.134 \\
\hline & $\mathrm{Pt} 7$ & 10.120 & -0.120 \\
\hline & Pt 8 & 10.124 & -0.124 \\
\hline \multirow[t]{8}{*}{$\mathrm{Pt}_{2} \mathrm{Ir}_{6}$} & Ir 1 & 8.942 & 0.058 \\
\hline & Ir 2 & 8.988 & 0.012 \\
\hline & Ir 3 & 8.933 & 0.067 \\
\hline & Ir 4 & 8.990 & 0.010 \\
\hline & Ir 5 & 8.957 & 0.043 \\
\hline & Ir 6 & 8.950 & 0.050 \\
\hline & $\mathrm{Pt} 7$ & 10.119 & -0.119 \\
\hline & Pt 8 & 10.124 & -0.124 \\
\hline \multirow[t]{8}{*}{$\mathrm{PtIr}_{7}$} & Ir 1 & 8.997 & 0.003 \\
\hline & Ir 2 & 9.003 & -0.003 \\
\hline & Ir 3 & 8.994 & 0.006 \\
\hline & Ir 4 & 8.954 & 0.046 \\
\hline & Ir 5 & 8.954 & 0.046 \\
\hline & Ir 6 & 8.989 & 0.011 \\
\hline & Ir 7 & 8.955 & 0.045 \\
\hline & Pt 8 & 10.153 & -0.153 \\
\hline \multirow[t]{8}{*}{$\mathrm{Ir}_{8}$} & Ir 1 & 8.984 & 0.016 \\
\hline & Ir 2 & 8.969 & 0.031 \\
\hline & Ir 3 & 9.021 & -0.021 \\
\hline & Ir 4 & 8.996 & 0.004 \\
\hline & Ir 5 & 9.025 & -0.025 \\
\hline & Ir 6 & 9.011 & -0.011 \\
\hline & Ir 7 & 8.998 & 0.002 \\
\hline & Ir 8 & 8.995 & 0.005 \\
\hline
\end{tabular}


Table S3. Local magnetic moments in the PAW sphere of both Pt and Ir atoms along with the spin population numbers in different orbitals are provided below.

\begin{tabular}{|c|c|c|c|c|c|}
\hline Clusters & Atoms (no.) & $\mathrm{s}\left(\mu_{\mathrm{B}}\right)$ & $\mathrm{p}\left(\mu_{\mathrm{B}}\right)$ & $\mathrm{d}\left(\mu_{\mathrm{B}}\right)$ & Total $\left(\mu_{\mathrm{B}}\right)$ \\
\hline \multirow[t]{8}{*}{$\mathrm{Pt}_{8}$} & $\mathrm{Pt} 1$ & 0.048 & 0.024 & 0.477 & 0.549 \\
\hline & Pt 2 & 0.048 & 0.024 & 0.477 & 0.549 \\
\hline & Pt 3 & 0.048 & 0.024 & 0.477 & 0.549 \\
\hline & $\mathrm{Pt} 4$ & 0.048 & 0.024 & 0.477 & 0.549 \\
\hline & Pt 5 & 0.018 & 0.009 & 0.172 & 0.198 \\
\hline & Pt 6 & 0.018 & 0.009 & 0.172 & 0.198 \\
\hline & $\operatorname{Pt} 7$ & 0.018 & 0.009 & 0.172 & 0.198 \\
\hline & Pt 8 & 0.018 & 0.009 & 0.172 & 0.198 \\
\hline \multirow[t]{8}{*}{$\mathrm{Pt}_{7} \mathrm{Ir}$} & Ir 1 & 0.045 & 0.016 & 0.538 & 0.598 \\
\hline & Pt 2 & -0.008 & 0.004 & 0.161 & 0.158 \\
\hline & Pt 3 & 0.008 & 0.002 & 0.309 & 0.320 \\
\hline & $\mathrm{Pt} 4$ & 0.012 & 0.009 & 0.356 & 0.376 \\
\hline & Pt 5 & 0.011 & 0.008 & 0.353 & 0.373 \\
\hline & $\operatorname{Pt} 6$ & 0.034 & 0.015 & 0.391 & 0.440 \\
\hline & $\mathrm{Pt} 7$ & 0.035 & 0.015 & 0.394 & 0.443 \\
\hline & Pt 8 & -0.008 & 0.001 & 0.110 & 0.103 \\
\hline \multirow[t]{8}{*}{$\mathrm{Pt}_{6} \mathrm{Ir}_{2}$} & Ir 1 & 0.003 & 0.000 & 0.429 & 0.432 \\
\hline & Ir 2 & 0.003 & 0.000 & 0.429 & 0.432 \\
\hline & Pt 3 & 0.019 & 0.012 & 0.443 & 0.475 \\
\hline & $\operatorname{Pt} 4$ & 0.019 & 0.012 & 0.443 & 0.475 \\
\hline & Pt 5 & 0.033 & 0.014 & 0.258 & 0.305 \\
\hline & Pt 6 & 0.019 & 0.012 & 0.443 & 0.475 \\
\hline & $\operatorname{Pt} 7$ & 0.019 & 0.012 & 0.443 & 0.475 \\
\hline & Pt 8 & 0.033 & 0.014 & 0.258 & 0.305 \\
\hline \multirow[t]{8}{*}{$\mathrm{Pt}_{5} \mathrm{Ir}_{3}$} & Ir 1 & 0.007 & 0.004 & 0.437 & 0.448 \\
\hline & Ir 2 & 0.009 & 0.004 & 0.427 & 0.440 \\
\hline & Ir 3 & 0.069 & 0.036 & 1.043 & 1.148 \\
\hline & $\mathrm{Pt} 4$ & 0.029 & 0.013 & 0.465 & 0.507 \\
\hline & Pt 5 & 0.033 & 0.014 & 0.248 & 0.296 \\
\hline & Pt 6 & 0.024 & 0.014 & 0.407 & 0.445 \\
\hline & $\mathrm{Pt} 7$ & 0.040 & 0.023 & 0.514 & 0.576 \\
\hline & Pt 8 & 0.032 & 0.014 & 0.230 & 0.276 \\
\hline \multirow[t]{8}{*}{$\mathrm{Pt}_{4} \mathrm{Ir}_{4}$} & Ir 1 & 0.047 & 0.036 & 0.684 & 0.767 \\
\hline & Ir 2 & 0.047 & 0.036 & 0.683 & 0.766 \\
\hline & Ir 3 & 0.047 & 0.036 & 0.683 & 0.766 \\
\hline & $\operatorname{Ir} 4$ & 0.047 & 0.036 & 0.683 & 0.766 \\
\hline & Pt 5 & 0.021 & 0.008 & 0.201 & 0.229 \\
\hline & Pt 6 & 0.021 & 0.008 & 0.201 & 0.229 \\
\hline & $\mathrm{Pt} 7$ & -0.018 & -0.008 & -0.122 & -0.148 \\
\hline & Pt 8 & -0.018 & -0.008 & -0.122 & -0.148 \\
\hline $\mathrm{Pt}_{3} \mathrm{Ir}_{5}$ & Ir 1 & 0.029 & 0.031 & 0.614 & 0.674 \\
\hline
\end{tabular}




\begin{tabular}{|c|c|c|c|c|c|}
\hline & Ir 2 & 0.030 & 0.031 & 0.621 & 0.682 \\
\hline & Ir 3 & 0.038 & 0.051 & 0.901 & 0.991 \\
\hline & Ir 4 & -0.003 & -0.005 & 0.019 & 0.011 \\
\hline & Ir 5 & 0.005 & 0.012 & 0.388 & 0.405 \\
\hline & Pt 6 & 0.012 & 0.018 & 0.396 & 0.426 \\
\hline & $\operatorname{Pt} 7$ & 0.015 & 0.009 & 0.390 & 0.414 \\
\hline & Pt 8 & 0.012 & 0.018 & 0.394 & 0.424 \\
\hline \multirow[t]{8}{*}{$\mathrm{Pt}_{2} \mathrm{Ir}_{6}$} & Ir 1 & 0.035 & 0.044 & 0.858 & 0.938 \\
\hline & Ir 2 & 0.005 & -0.002 & 0.027 & 0.031 \\
\hline & Ir 3 & 0.018 & 0.031 & 0.686 & 0.735 \\
\hline & Ir 4 & 0.014 & 0.015 & 0.270 & 0.300 \\
\hline & Ir 5 & 0.035 & 0.044 & 0.859 & 0.938 \\
\hline & Ir 6 & 0.018 & 0.030 & 0.684 & 0.733 \\
\hline & $\mathrm{Pt} 7$ & 0.018 & 0.014 & 0.404 & 0.436 \\
\hline & Pt 8 & 0.021 & 0.016 & 0.477 & 0.513 \\
\hline \multirow[t]{8}{*}{$\mathrm{PtIr}_{7}$} & Ir 1 & 0.003 & 0.011 & 0.298 & 0.311 \\
\hline & Ir 2 & -0.003 & -0.013 & -0.081 & -0.097 \\
\hline & Ir 3 & 0.003 & 0.011 & 0.297 & 0.310 \\
\hline & Ir 4 & 0.034 & 0.024 & 0.585 & 0.644 \\
\hline & Ir 5 & 0.035 & 0.024 & 0.586 & 0.645 \\
\hline & Ir 6 & 0.003 & 0.011 & 0.296 & 0.310 \\
\hline & Ir 7 & 0.035 & 0.024 & 0.585 & 0.644 \\
\hline & Pt 8 & 0.018 & 0.020 & 0.444 & 0.482 \\
\hline \multirow[t]{8}{*}{$\mathrm{Ir}_{8}$} & Ir 1 & 0.000 & 0.000 & -0.002 & -0.003 \\
\hline & Ir 2 & 0.000 & 0.000 & 0.001 & 0.001 \\
\hline & Ir 3 & -0.001 & -0.001 & -0.005 & -0.007 \\
\hline & Ir 4 & 0.001 & 0.001 & 0.007 & 0.008 \\
\hline & Ir 5 & 0.000 & 0.000 & 0.000 & 0.000 \\
\hline & Ir 6 & 0.001 & 0.000 & 0.005 & 0.006 \\
\hline & Ir 7 & 0.000 & 0.000 & -0.001 & -0.001 \\
\hline & Ir 8 & -0.001 & -0.001 & -0.005 & -0.006 \\
\hline
\end{tabular}




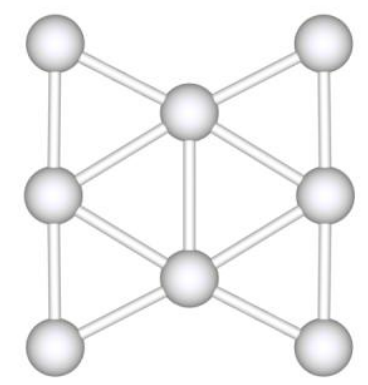

tetra-capped rhombus

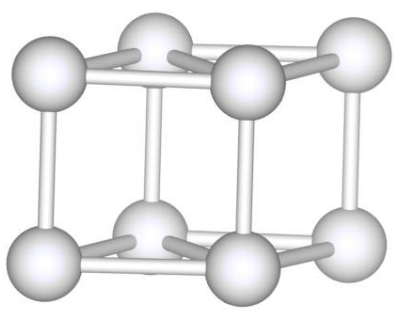

rhombic prism

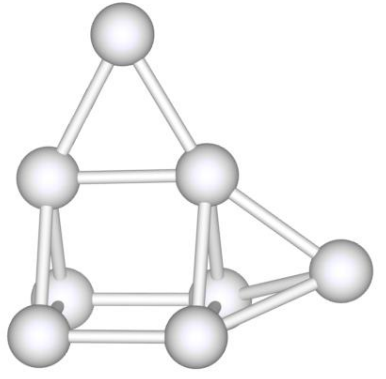

bicapped trigonal prism

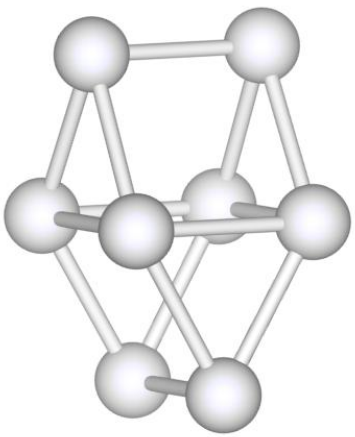

orthogonal prism

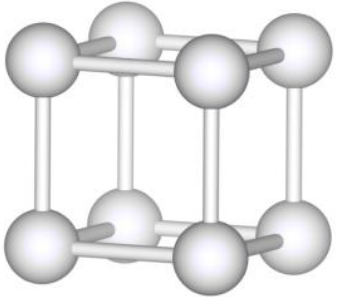

cube

Figure S1. Most favorable geometrical structures of the low-lying isomers for different compositions at a given size. 